\title{
Formulating chemical fugacity for general circulation models
}

\author{
$\underline{\text { Michael L. Bates }}^{\text {a }}$, Roger A. Cropp ${ }^{\text {a }}$ and Darryl W. Hawker ${ }^{\text {a }}$ \\ ${ }^{a}$ Griffith School of Environment, Griffith University, Brisbane, QLD 4111, Australia \\ Email:m.bates@griffith.edu.au
}

\begin{abstract}
Fugacity is identical to the partial pressure of an ideal gas in air, however, for real gases fugacity is much more accurate than partial pressure in chemical equilibrium calculations. Since the first use of environmental chemical fugacity models in the late 1970's they have been utilised extensively in the study of the local and global partitioning and distribution of chemicals in the environment. Global climate models are becoming an increasingly important tool in understanding the effects of climate change. Climate models are now being applied to understanding the distribution of persistent organic pollutants (POPs). POPs are a class of toxic chemicals that can bioconcentrate, bioaccumulate and biomagnify. Chemical fugacity is being used to calculate the inter-phase fluxes, however, the intra-phase fluxes are still being calculated using chemical concentration.

Here we formulate equations that are suitable for the solution of intra-phase chemical fluxes in general circulation models using a chemical fugacity approach. Using a relaxation experiment in a one-dimensional (in the vertical) ocean, we examine how the the fugacity approach compares to the standard approach of using chemical concentration. We find that there are two major differences. Firstly, in the equilibrium calculation the standard method homogenises the concentration in the water column, while the fugacity approach has a depth dependent concentration with a uniform fugacity. Secondly, the timescale of equilibration is different, even though the turbulent diffusivity is the same in both simulations. These results suggest that climate models that model POPs should use a fugacity approach for the calculation of intra-phase fluxes, and not only inter-phase fluxes.
\end{abstract}

Keywords: General circulation model, fugacity modelling, persistent organic pollutants 


\section{INTRODUCTION}

Persistent organic pollutants (POP) are toxic, mostly anthropogenic chemicals that have become ubiquitous global pollutants. They can undergo long range environmental transport through both atmospheric and oceanic circulation. Despite ongoing regulation on emissions, the global environment now carries a chemical burden which potentially represents a significant threat to human and wildlife health. Once these toxic chemicals have been released they cannot be controlled, hence environmental management of the chemical threat must come from a robust understanding of their environmental behaviour and fate.

Many POPs are lipophilic which leads to their bioaccumulation and biomagnification in the food web. Some POPs are internationally regulated by the Stockholm Convention (UNEP, 2001). The continued emergence of new POPs and changing environmental conditions (e.g. climate change) provides many challenges to their ongoing regulation (Armitage et al., 2011; Bengtson Nash, 2011).

Modelling the environmental fate and behaviour of chemicals using their fugacity has been used to great effect for well over three decades (Mackay and Paterson, 1981). Fugacity models have become an integral tool for scientists to understand the transport and distribution of POPs (MacLeod et al., 2010).

Coupled general circulation models (GCMs) are used to simulate climate, especially future climate. Given the global nature of POPs and the uncertainty of the effects of climate change on that distribution, GCMs are a potentially extremely useful tool in the study of future global distribution of POPs. Indeed, Guglielmo et al. (2009) have used GCMs to model the global fractionation of POPs and have already found some interesting results, one of which is that the deep ocean, which presently acts as a sink of POPs may start to act as a secondary source of POPs (Stemmler and Lammel, 2013).

These previous studies have used a fugacity approach to calculate the inter-phase fluxes of POPs, however, they have not used a fugacity approach for the intra-phase transport. It is unclear whether there is an appreciable difference between using a concentration gradient (as is done in GCMs for chemical tracers) or a fugacity gradient (as is done in a fugacity approach) to calculate diffusive fluxes makes an appreciable difference or not to the model's solution. The objective of this paper is to show how to formulate finite volume equations of chemical fugacity for both intra- and inter-phase transport. Furthermore, we investigate whether using a fugacity approach can make an appreciable difference to the simulated distribution of POPs in the ocean.

\section{Chemical TRACERS IN GCMS}

In this section we detail the treatment of chemical tracers in GCMs and rationalise that with the formulation of chemical fugacity models. We focus on the ocean, however, analogous arguments hold for the atmosphere too.

The mass of a chemical tracer in a finite volume (e.g. a grid cell) is given by the integral of the concentration multiplied by the density of seawater

$$
M=\iiint_{V} \rho \mathcal{C} \mathrm{d} \mathbf{x}
$$

where $M$ is the mass of the chemical $(\mathrm{kg}), \rho$ is the density of seawater $\left(\mathrm{kg} \mathrm{m}^{-3}\right), \mathcal{C}$ is the tracer concentration (with units of mass of chemical per mass of seawater, $\mathrm{kg} \mathrm{kg}^{-1}$ ), $V$ is the finite volume being integrated (e.g. the grid cell), and $\mathbf{x}$ is a vector three-space (where $\hat{\mathbf{x}}, \hat{\mathbf{y}}$, and $\hat{\mathbf{z}}$ are orthogonal unit vectors to describe physical space). Here, we allow the volume of integration to vary with time, $V=V(t)$.

To find the rate of change of chemical in $V$ we take the material derivative of Equation (1) and use Reynolds Transport Theorem

$$
\frac{\mathrm{d} M}{\mathrm{~d} t}=\frac{\mathrm{d}}{\mathrm{d} t} \iiint_{V} \rho \mathcal{C} \mathrm{d} \mathbf{x}=\iiint_{V} \frac{\partial \rho \mathcal{C}}{\partial t} \mathrm{~d} \mathbf{x}+\iint_{\partial V} \rho \mathcal{C} \mathbf{v}^{b} \cdot \hat{\mathbf{n}} \mathrm{d} S,
$$

where $\partial V$ is the closed boundary of of the volume, $\mathbf{v}^{b}$ is the velocity of the closed boundary, $\hat{\mathbf{n}}$ is the outward pointing normal vector of $V$, and $S$ is the surface being integrated.

We can quantify the change in concentration with a source/sink term and a flux divergence term

$$
\frac{\partial \rho \mathcal{C}}{\partial t}=\rho \mathcal{S}-\nabla \cdot \mathbf{J}
$$


where $\mathcal{S}$ are tracer sources/sinks $\left(\mathrm{s}^{-1}\right), \nabla=\left(\frac{\partial}{\partial x}, \frac{\partial}{\partial y}, \frac{\partial}{\partial z}\right)$ is the gradient operator, and $\mathbf{J}$ is the tracer flux $\left(\mathrm{m} \mathrm{s}^{-1}\right)$. Integrating Equation (3) yields

$$
\begin{aligned}
\iiint_{V} \frac{\partial \rho \mathcal{C}}{\partial t} \mathrm{~d} \mathbf{x} & =\iiint_{V} \rho \mathcal{S} \mathrm{d} \mathbf{x}-\iiint_{V} \nabla \cdot \mathbf{J} \mathrm{d} \mathbf{x} \\
& =\iiint_{V} \rho \mathcal{S} \mathrm{d} \mathbf{x}-\iint_{\partial V} \mathbf{J} \cdot \hat{\mathbf{n}} \mathrm{d} S
\end{aligned}
$$

where the divergence theorem (e.g. Aris, 1962) has been used to describe the change in concentration in terms of the flux through the boundary of the volume. This simplifies the problem such that we need only concern ourselves with the flux across the boundary of the volume, rather than at every point in the volume.

The fluxes in Equation (4) can be divided into two broad types: diffusive fluxes and advective fluxes. Advective fluxes are fluxes that are related to transport processes (e.g. wind, water flow, precipitation) and are related to the velocity of the advective process

$$
\mathbf{J}^{a}=\rho \mathcal{C} \mathbf{v},
$$

where $\mathbf{v}$ is the velocity (or effective velocity) of the advective process.

Diffusive parameterisations act to equilibrate the chemical potential and are used to describe the effect of processes such as molecular or turbulent diffusion. In GCMs the gradient of the chemical potential is assumed to be proportional to the gradient of the concentration, and thus diffusive processes are parameterised as a flux proportional to the concentration gradient,

$$
\mathbf{J}^{d}=-\rho \boldsymbol{\kappa} \cdot \nabla \mathcal{C}
$$

where $\kappa$ is the diffusivity tensor (a rank two tensor) with units of $\mathrm{m}^{2} \mathrm{~s}^{-1}$.

Combining Equations (1) to (6) gives a general expression for the tracer budget of a grid cell in a GCM,

$$
\frac{\mathrm{d} M}{\mathrm{~d} t}=\iiint_{V} \rho \mathcal{S} \mathrm{d} \mathbf{x}-\iint_{\partial V}\left(\rho \mathcal{C} \mathbf{v}^{s}-\rho \boldsymbol{\kappa} \cdot \nabla \mathcal{C}\right) \cdot \hat{\mathbf{n}} \mathrm{d} S
$$

where we have written the dia-surface velocity (i.e. the velocity of the flow relative to the velocity of the boundary of $V$ ) as $\mathbf{v}^{s}=\mathbf{v}^{b}-\mathbf{v}$.

\section{USING FUGACITY TO CALCULATE DIFFUSIVE FLUXES}

Fugacity is related to the concentration as

$$
\rho \mathcal{C}=M_{m} Z f
$$

where $Z$ is the fugacity capacity $\left(\mathrm{mol} \mathrm{m}^{-3} \mathrm{~Pa}^{-1}\right), f$ is the fugacity $(\mathrm{Pa})$, and $M_{m}$ is the molar mass of the chemical $\left(\mathrm{kg} \mathrm{mol}^{-1}\right)$. As discussed in Section 1, diffusive processes will tend to equilibrate the fugacity and thus the diffusive flux can be related to the fugacity gradient

$$
\mathbf{J}^{d}=-M_{m} Z \boldsymbol{\kappa} \cdot \nabla f
$$

This gives an alternative expression to Equation (7) for the budget of a grid cell,

$$
\frac{\mathrm{d} M}{\mathrm{~d} t}=\iiint_{V} \rho \mathcal{S} \mathrm{d} \mathbf{x}-\iint_{\partial V}\left(\rho \mathcal{C} \mathbf{v}^{s}-M_{m} Z \boldsymbol{\kappa} \cdot \nabla f\right) \cdot \hat{\mathbf{n}} \mathrm{d} S,
$$

which could be used in in GCMs for calculating the budget of a grid cell, where the diffusive fluxes (e.g. turbulent diffusion) are calculated using chemical fugacity.

Substituting Equation (8) into Equation (6) shows that the diffusive fluxes calculated using the concentration gradient is equivalent to the fluxes calculated using the fugacity gradient, Equation (9), if the fugacity capacity is constant.

The fugacity capacity of water is temperature dependent and is given by the inverse of Henry's Law constant,

$$
Z=H^{-1} \text {. }
$$


M.L. Bates et al., Formulating chemical fugacity for general circulation models

Table 1. Parameter values used in the model of turbulent diffusion of HCB through the water column.

\begin{tabular}{|lr|lr|}
\hline Parameter & Value & Parameter & Value \\
\hline$T^{\text {ref }}$ & $298 \mathrm{~K}$ & $\rho$ & $1032 \mathrm{~kg} \mathrm{~m}^{-3}$ \\
$M_{m}$ & $0.2848 \mathrm{~kg} \mathrm{~mol}^{-1}$ & $\Delta U_{A W}$ & $50490 \mathrm{~J} \mathrm{~mol}^{-1}$ \\
$R$ & $8.314 \mathrm{~Pa} \mathrm{~m}^{3} \mathrm{~mol}^{-1} \mathrm{~K}^{-1}$ & $H^{\text {ref }}$ & $172 \mathrm{~Pa} \mathrm{~m}^{3} \mathrm{~mol}^{-1}$ \\
\hline
\end{tabular}

Henry's Law constant varies with temperature and, therefore, we adjust for temperature using a van 't Hoff correction

$$
H=H^{\mathrm{ref}} \exp \left(-\frac{\Delta U_{A W}}{R}\left[\frac{1}{T_{W}}-\frac{1}{T^{\mathrm{ref}}}\right]\right) .
$$

Here $H^{\text {ref }}$ is Henry's Law constant at the reference temperature $T^{\text {ref }}, \Delta U_{A W}$ is the difference in internal energy of the chemical between air and water, $R$ is the ideal gas constant, and $T_{W}$ is the temperature of the water.

When modelling environmental transport of chemicals a reasonable question to ask is, does the difference between the diffusive flux calculated using Equations (6) and (9) make a meaningful difference? If the fugacity capacity is approximately constant in the domain (i.e. if the domain has a uniform temperature) then the two formulations are mathematically equivalent and there is no difference. On the other hand, if the fugacity capacity is not uniform there may be a non-trivial difference in the calculation of diffusive fluxes.

To explore whether using the fugacity gradient will provide a significantly different solution to that obtained using concentration gradient, we constructed a one dimensional (in the vertical) model of the ocean and solved for the POP mass at each vertical level using each of the two methods. We use chemical parameters of the ubiquitous POP hexachlorobenzene (HCB), the values for which can be found in Table 1. The numerical solutions were obtained using the Dormand and Prince (1980) 4(5) Runge-Kutta method as implemented in the ODE module of SciPy (Oliphant, 2007).

The model ocean is $5500 \mathrm{~m}$ deep and consists of 32 levels from $10 \mathrm{~m}$ thick at the surface to $500 \mathrm{~m}$ thick at the ocean bottom. The temperature, used to calculate the fugacity capacity, is shown in Figure 1. It was found by taking the level average of the annual mean World Ocean Atlas (Locarnini et al., 2010) between $50^{\circ} \mathrm{S}$ and $50^{\circ} \mathrm{N}$.

The experiment was a relaxation experiment, where an initial concentration of HCB of $5 \times 10^{-16} \mathrm{~kg} \mathrm{~kg}^{-1}$ was set in top grid cell (i.e. the top $10 \mathrm{~m}$ ) of the ocean and there was no exchange with any other phase (e.g. atmosphere or sediment). The only physical process to affect the concentration of HCB was vertical turbulent diffusivity. The depth dependent diffusivity profile was the arctagent profile of Bryan and Lewis (1979), which is shown in grey in Figure 1

The first experiment used the concentration gradient to calculate the fluxes and solve for the HCB mass at each level,

$$
\frac{\mathrm{d} M}{\mathrm{~d} t}=\iint_{\partial V} \rho \kappa \frac{\partial \mathcal{C}}{\partial z} \mathrm{~d} x \mathrm{~d} y
$$

while the second experiment used the fugacity gradient to solve for the HCB mass at each level

$$
\frac{\mathrm{d} M}{\mathrm{~d} t}=\iint_{\partial V} M_{m} Z \kappa \frac{\partial f}{\partial z} \mathrm{~d} x \mathrm{~d} y .
$$

Time series of the evolution of concentration at each level is shown in Figure 2. The lines that originate at $5 \times 10^{-16} \mathrm{~kg} \mathrm{~kg}^{-1}$ is the concentration of the top grid cell, while the lines originating at the origin is the concentration in the other grid cells.

The dashed lines show the concentration for the experiment using the concentration gradient. Since the diffusive flux depends on the concentration gradient, the flux will act to homogenise the concentration to $9.091 \times 10^{-19} \mathrm{~kg} \mathrm{~kg}^{-1}$ (not shown). The solid lines, however, are not converging to the same concentration but instead are converging to a uniform fugacity of $3.277 \times 10^{-14} \mathrm{~Pa}$ (not shown). At this equilibrium fugacity the depth dependent fugacity capacity leads a concentration that varies from $6.651 \times 10^{-20} \mathrm{~kg} \mathrm{~kg}^{-1}$ in the top 


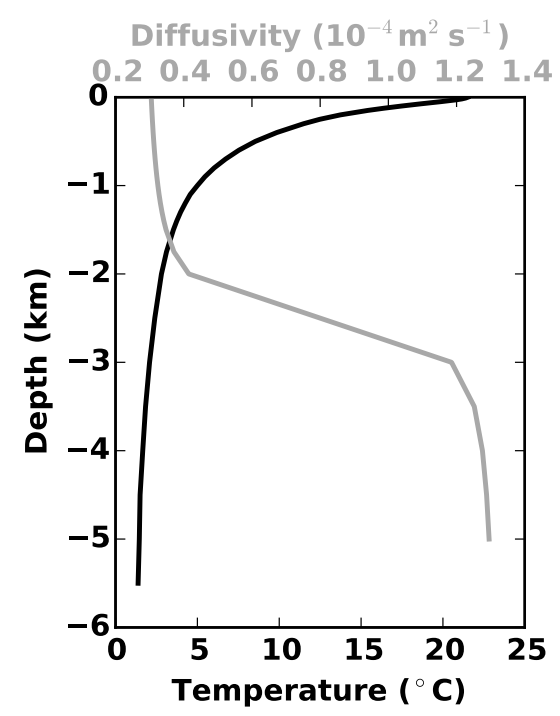

Figure 1. The temperature (black) and diffusivity (grey) profile used in the model.

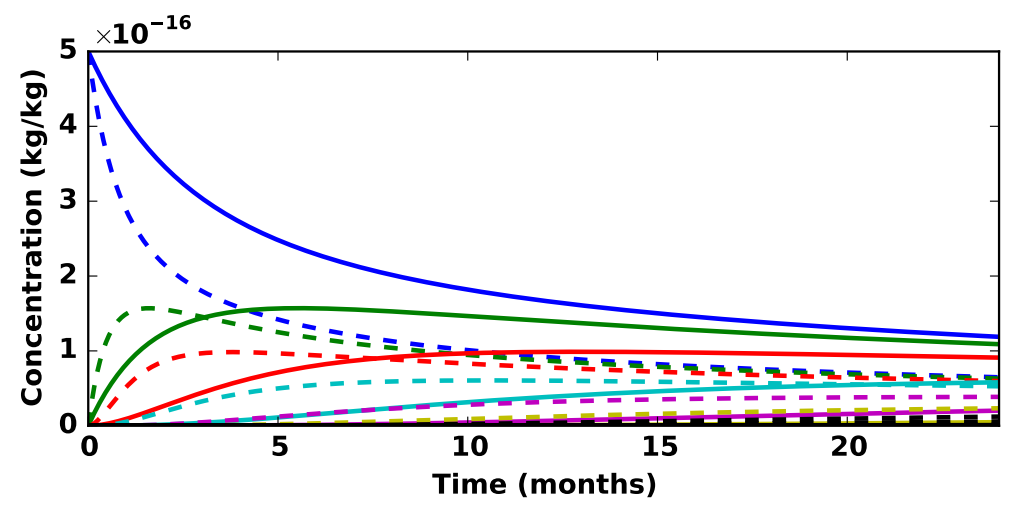

Figure 2. The evolution of concentration at each level in a model that uses concentration gradient (dashed lines) and one that uses the fugacity gradient (solid lines) to calculate the diffusive flux of HCB through the water column. Colours indicate corresponding grid cells. 
grid cell to $2.994 \times 10^{-19} \mathrm{~kg} \mathrm{~kg}^{-1}$ in the bottom grid cell. To reach these equilibrium values, however, takes thousands of simulated years. Nevertheless, Figure 2 shows that the difference in approaches has a significant effect on the transient solutions on monthly and annual timescales.

Another important difference between the two experiments is that the timescale of change is also depth dependent; the rate of change of concentration in the upper two grid cells is slower using a fugacity approach, but is faster for deeper cells. These results show that, given the same initial conditions, the evolution and final distribution of HCB in the water column is quite sensitive to whether the concentration gradient or the fugacity gradient is used to calculate the vertical diffusive flux of HCB in the ocean.

\section{Conclusions}

We have shown how a fugacity model for the calculation of diffusive fluxes of POPs would be formulated for a GCM. A fugacity approach has been used for decades in the calculation of inter-phase fluxes of POPs, however, the use of a fugacity approach for the calculation of intra-phase fluxes has, to our knowledge, not been investigated.

To ascertain whether a fugacity approach would have an impact on the model solution we used a simple onedimensional model of the ocean water column. This model demonstrated that the difference in the modelled distribution of HCB in the water column is non-trivial. This result has implications for the modelling of the global distribution of POPs.

Using a coupled GCM Stemmler and Lammel (2013) showed that the ocean may act as a secondary source of POPs. They produced vertical profiles of the concentration of a number of POPs. Our results indicate that because of the temperature dependence of the fugacity capacity, a fugacity implementation of the diffusive fluxes may affect the timescales of diffusion and, ultimately, the depth dependence of the POP concentration.

If the fugacity capacity is constant the diffusive fluxes calculated using the concentration gradient and the fugacity gradient are mathematically identical. Since the fugacity capacity of POPs are temperature dependent the difference between a concentration and fugacity implementation is only appreciable in regions of relatively large temperature gradients. This explains why the vertical distribution of HCB over the ocean thermocline is sensitive to whether the concentration gradient or fugacity gradient is used to calculate the turbulent fluxes.

In models, such as many regional models, where the temperature of the atmosphere and ocean is relatively homogeneous, the sensitivity of the solution to a concentration or fugacity implementation is likely quite trivial. However, as we have demonstrated here, models with strong temperature gradients, such as of the ocean thermocline or polar fronts, the use of a fugacity implementation for the intra-phase calculation of turbulent fluxes of POPs will affect those fluxes and thus, the modelled distribution of POPs.

\section{ACKNOWLEDGEMENT}

This project was made possible through funding from Australian Antarctic Science grant number 4121. Plotting was done using matplotlib (Hunter, 2007).

\section{REFERENCES}

Aris, R. (1962). Vectors, Tensors and the Basic Equations of Fluid Mechanics. Dover Publications.

Armitage, J. M., C. L. Quinn, and F. Wania (2011, June). Global climate change and contaminants-an overview of opportunities and priorities for modelling the potential implications for long-term human exposure to organic compounds in the Arctic. J. Environ. Monit. 13(6), 1532-46.

Bengtson Nash, S. M. (2011, March). Persistent organic pollutants in Antarctica: current and future research priorities. J. Environ. Monit. 13(3), 497-504.

Bryan, K. and L. J. Lewis (1979). A water mass model of the World Ocean. J. Geophys. Res. 84(C5), 2503.

Dormand, J. and P. Prince (1980, March). A family of embedded Runge-Kutta formulae. J. Comput. Appl. Math. 6(1), 19-26.

Guglielmo, F., G. Lammel, and E. Maier-Reimer (2009, September). Global environmental cycling of gamma$\mathrm{HCH}$ and DDT in the 1980s-a study using a coupled atmosphere and ocean general circulation model. Chemosphere 76(11), 1509-17. 
M.L. Bates et al., Formulating chemical fugacity for general circulation models

Hunter, J. D. (2007, May). Matplotlib: A 2D Graphics Environment. Comput. Sci. Eng. 9(3), 90-95.

Locarnini, R. A., A. V. Mishonov, J. I. Antonov, T. P. Boyer, H. E. Garcia, O. K. Baranova, M. M. Zweng, and D. R. Johnson (2010). World Ocean Atlas 2009, Volume 1: Temperature. Washington D.C.: U.S. Government Printing Office.

Mackay, D. and S. Paterson (1981, October). Calculating fugacity. Environ. Sci. Technol. 15(9), 1006-14.

MacLeod, M., M. Scheringer, T. E. McKone, and K. Hungerbuhler (2010, November). The State of Multimedia Mass-Balance Modeling in Environmental Science and Decision-Making. Environ. Sci. Technol. 44(22), 8360-8364.

Oliphant, T. E. (2007, May). Python for Scientific Computing. Comput. Sci. Eng. 9(3), 10-20.

Stemmler, I. and G. Lammel (2013, April). Evidence of the return of past pollution in the ocean: A model study. Geophys. Res. Lett. 40(7), 1373-1378.

UNEP (2001). Stockholm Convention on Persistent Organic Pollutants. Technical report, United Nations Environment Program, Stockholm. 